\title{
Cancer Progression
}

National Cancer Institute

\section{Source}

National Cancer Institute. Cancer Progression. NCl Thesaurus. Code C19987.

A pathologic process in which alterations at the molecular level result in a more

aggressive cytologic and phenotypic profile and clinical course of a malignant neoplasm. 